\title{
Resistencia a la tracción diametral in vitro de cinco cementos dentales usados como cementantes de puentes y coronas en prótesis fijas
}

\author{
In vitro diametral tensile strength of five dental cements used as bridge and crown cement in fixed prostheses \\ Elizabeth Chávez Sánchez 1,a,b, Wilber Jiménez Mendoza ${ }^{2,, d, e, ~ K e l l y ~ M a l p a r t i d a ~ V a l d e r r a m a ~}{ }^{1, b}$, \\ Carolina Soto Carrión ${ }^{3, \mathrm{~d}, \mathrm{e}, \mathrm{f}, \mathrm{g}}$
}

\begin{abstract}
RESUMEN
Objetivo: El propósito de la investigación fue evaluar la resistencia a la tracción diametral in vitro de cinco cementos dentales: BisCem, Duolink, Ketac Cem, Meron, Allcem, cuatro adhesivos convencionales y un autoadhesivo polimerizado químicamente utilizados como cementantes de puentes y coronas en prótesis fijas. Materiales y métodos: Se prepararon 100 especímenes conformados por cementos: BisCem, Duolink, Ketac Cem, Meron, Allcem, los cuales se dividieron aleatoriamente en 5 grupos de 20 especímenes cada grupo sujetas a fotopolimerización y auto polimerización de $8 \mathrm{~mm}$ de diámetro y $5 \mathrm{~mm}$ de alto, con $2 \mathrm{~mm}$ de distancia aproximadamente de la lámpara para la polimerización, se utilizó un formador de probetas de polietileno de alta densidad estandarizado, el tipo de muestreo es no probabilístico. Se sometieron a los especímenes de cada grupo a la prueba de tracción utilizando una máquina de ensayo universal (zwickiLine by Zwick/Roell), hasta lograr el fracaso del cemento, sometidas a una fuerza continua de $500 \mathrm{~kg}$, con una velocidad de desplazamiento fija de $1 \mathrm{~cm} / \mathrm{min}$. Resultados: La resistencia promedio a la tracción diametral de probetas del cemento BisCem fue de $38,75 \pm 11,56 \mathrm{MPa}$, del cemento Duolink fue 64,30 MPa, del cemento Meron 57,14 MPa, del cemento Ketac Cem es de 32,23 MPa, del cemento BisCem (38,753 MPa) y el cemento Ketac Cem (39,233 MPa). El cemento dental Duolink es aquel que presenta mayor resistencia a la tracción diametral de probetas en comparación a Meron (57,137 MPa) que es su similar. La tabulación y el análisis han sido sometidos a los test de Shapiro Wilk, análisis de varianza ANOVA y la prueba de Tuckey los valores de cada muestra se distribuyen normalmente existiendo diferencias significativas entre los grupos estudiados. Conclusiones: Las probetas del cemento de resina adhesiva Duolink evidenció una mayor resistencia a la tracción diametral con mejores valores significativos de resistencia a diferencia de los cementos Ketac Cem, Allcem, Meron y BisCem.
\end{abstract}

PALABRAS CLAVE: Cemento dental, prótesis dental, cementación, corona dental.

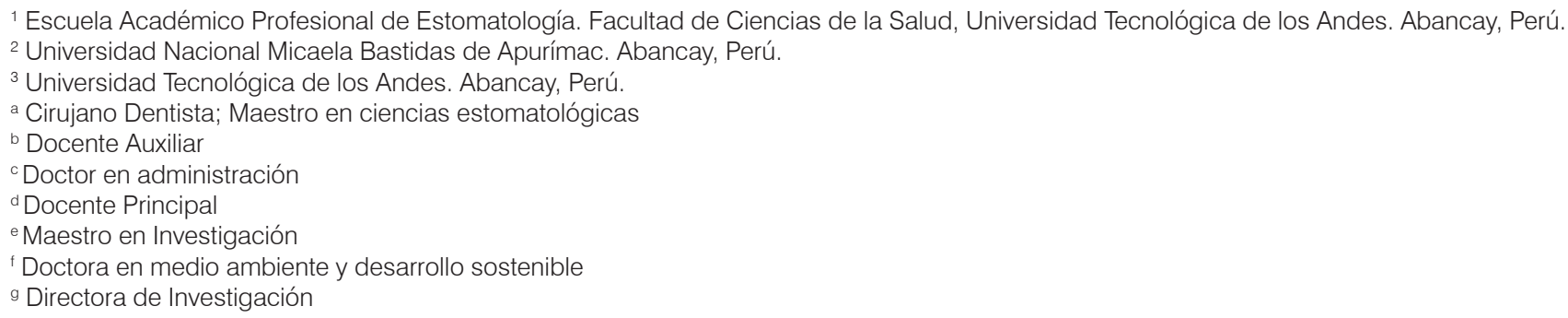




\section{SUMMARY}

Objetive: The purpose of the research was to evaluate the in vitro diametral tensile strength of five dental cements: BisCem, Duolink, Ketac Cem, Meron, Allcem, four conventional adhesives and a chemically polymerized self-adhesive used as bridge cements and crowns in fixed dentures. Materials and methods: 100 specimens consisting of cements were prepared: BisCem, Duolink, Ketac Cem, Meron, Allcem, which were randomly divided into 5 groups of 20 specimens each group subject to photopolymerization and self-polymerization of $8 \mathrm{~mm}$ in diameter and $5 \mathrm{~mm}$ high, with an approximate distance of $2 \mathrm{~mm}$ from the lamp for polymerization, a standardized high density polyethylene specimen was used, the type of sampling is not probabilistic. The specimens of each group were subjected to tensile testing using a universal testing machine (zwickiLine by Zwick / Roell), until the failure of the cement was achieved, subjected to a continuous force of $500 \mathrm{~kg}$, with a fixed displacement speed of $1 \mathrm{~cm} / \min$ Results: The average tensile strength of diametral tensile specimens of BisCem cement was $38.75 \pm 11.56 \mathrm{MPa}$, Duolink cement was $64.30 \mathrm{MPa}$, Meron cement 57.14 MPa, Ketac Cem cement was 32.23 $\mathrm{MPa}$, BisCem cement (38.753 MPa ) and Ketac Cem cement (39,233 MPa). Duolink dental cement is the one with the highest tensile strength of diametral specimens compared to Meron $(57,137 \mathrm{MPa})$, which is similar. The tabulation and the analysis have been submitted to the Shapiro Wilk test, ANOVA analysis of variance and the Tuckey test. The values of each sample are normally distributed with significant differences between the studied groups. Conclusions: Duolink adhesive resin cement specimens showed a higher diametral tensile strength with better significant strength values unlike Ketac Cem, Allcem, Meron and BisCem cements.

KEYWORDS: Dental cement, dental prothesis, cementation, dental crown.

\section{INTRODUCCION}

En los últimos años la Estomatología y la rama de la prótesis fija han venido evolucionadas drásticamente gracias al desarrollo de la ciencia, la tecnología y la innovación. En el caso de las prótesis fijas existen técnicas de su confección y de su cementación. La prótesis fija definitiva cementada sobre metal tiene un gran parecido con la prótesis cementada sobre diente naturales; muchas de las ventajas que poseen las prótesis cementadas, así como los procedimientos de elaboración que corresponden a la sistemática de elaboración que se emplea en el diente natural. En cambio, a nivel clínico algunos de los protocolos de cementación que se siguen corresponden a protocolos basados en el concepto de restauración provisional.

Las prótesis fijas han representado una opción predecible en el tratamiento del edentulismo parcial, la cual presenta limitantes. Las prótesis fijas sobre reconstrucciones de los pilares no son susceptibles a caries y podrían aumentar la sobrevivencia de este tipo de prótesis. Polesel $^{1}$, manifiesta que gracias a la evolución de la adherencia y de los nuevos materiales de restauración a base de composite, con propiedades mecánicas similares a los de los tejidos dentarios, ofrecen al odontólogo la opción de realizar una restauración indirecta conservadora, capaz de sellar, re- forzar y proteger el diente, evitando sacrificar tejido dental (1).

La principal causa de fracaso es la pérdida de retención de la restauración con la desinsersión de la misma. La unidad restauración - agente cementante en medio oral se encuentra sometido a fuerzas intraorales complejas para lo cual el cemento debe tener unos valores de resistencia altos, entre ellas la resistencia a fuerzas de tracción de forma directa o indirecta que provocaría su desinserción de la cavidad. Los cementos resinosos se han establecido como el material preferido para la fijación de restauraciones indirectas estéticas en los últimos años. Esto ha ocurrido debido a los avances en propiedades físicas, mejoras en las características estéticas, manejo y técnica cada vez más (2).

Rivas $^{3}$ manifestó que el ensayo de tracción ayuda en determinar las propiedades mecánicas de los materiales específicamente de sus características de resistencia y deformación, permitiendo conocer el límite de elasticidad, la carga máxima que puede soportar y la resistencia estática del material en estudio, las probetas calibradas utilizadas para la experimentación se diseñan para ser sujetadas en la máquina universal de ensayos y para que el punto de fractura se produzca en un punto establecido.

Las pruebas de tracción se realizan en una máquina 
universal de ensayos calibrada previamente, el ensayo finaliza cuando existe un desprendimiento o separación del material estudiado, el resultado se mide en Newton, unidad de fuerza que aplicada a una masa produce una aceleración (3).

El ensayo de tracción de un material consiste en someter a una probeta normalizada a un esfuerzo axial de tracción creciente hasta que se produce la rotura de la probeta. Este ensayo mide la resistencia de un material a una fuerza estática o aplicada lentamente; las velocidades de deformación en un ensayo de tensión suelen ser muy pequeñas (4).

El termociclado tiene como objetivo simular el ambiente de la cavidad bucal utilizando variaciones de temperatura utilizando agua entre 5 a $55^{\circ} \mathrm{C}$ para sumergir las muestras de estudio, y con ello lograr un envejecimiento en la interfaz adhesiva y el material restaurador en un periodo corto de tiempo, según el protocolo estándar ISO TR 11450 de 1994 se recomienda 500 ciclos para lograr el envejecimiento, sin embargo estudios más recientes revelan que es necesario una aumentar la cantidad de ciclos para lograr un envejecimiento verdadero aunque la cantidad es aun debatida (5).

El envejecimiento se produce debido al agua caliente que acelera la hidrólisis de los componentes del material adhesivo, además del estrés causado por la expansión y contracción del material al ser sometidos a cambios de temperatura continuos, esto se debe a que posee un coeficiente de expansión térmica mucho mayor en relación a las estructuras dentarias, provocando un envejecimiento acelerado (5).

Los cementos resinosos a pesar de tener el mismo componente básico que las resinas compuestas, poseen una menor concentración de partículas de relleno para mantener una viscosidad adecuada para el cementado y garantizar un espesor de película adecuado (6).

Los cementos resinosos, dependiendo de su mecanismo de activación, se pueden clasificar en: cementos de autocurado si inician su reacción por métodos químicos; fotocurado si es mediante luz y dual si polimerizan por ambos métodos $(7,8)$.

Hacia los años 90s, Van Dijken y Pallesen, mencionan que la utilización de resina restaurativa como medio cementante, tiene como ventaja un margen de alto contenido de relleno, que no se desgasta, es fácil de retirar los excesos previo a su polimerización, capaz de rellenar cualquier pequeño defecto, de fácil de pulido y terminado; pero con la desventaja de lograr obtener una capa de resina lo suficientemente fluida y delgada para no alterar su total asentamiento y así permitir su completa polimerización (9).

Palacio, el 2014 evaluó la resistencia flexural de 45 bloques de resina compuesta (Tetric N-Ceram Ivoclar) de $2 \mathrm{~mm}$ de ancho y $2 \mathrm{~mm}$ de espesor precalentada a $60^{\circ} \mathrm{C}$, en diferentes tiempos $(10,20,30,40,50$ y 60 minutos), donde el incremento de la temperatura produce, un aumento en la resistencia a la flexión, la misma que puede ser utilizada luego de ser sometida a múltiples cambios térmicos (10).

Pozo y García el 2015, evaluaron la resistencia a la tracción de tres sistemas de cementación resina fluida Filtek $^{\mathrm{TM}}$ Z350XT, cemento de curado dual RelyXTM ARC 3M ESPE y cemento autoadhesivo RelyXTM U200 3M ESPE, para cerómeros, obteniendo como resultado: para la resina fluida un valor de 2,25 MPa, para el cemento de curado dual de $1,46 \mathrm{MPa}$, mientras que, para el cemento autoadhesivo de $3,8 \mathrm{MPa}$; presentando este último un mejor comportamiento clínico (11).

La importancia de un buen sellado marginal, a través de la utilización de resina precalentada, la que demostró su eficiencia en la cementación de restauraciones indirectas evitando la formación de grietas al lograr un contacto perfecto, sin microfiltraciones en las superficies tanto distal, mesial y oclusal. Con ello se logra tratamientos de larga duración con resultados satisfactorios tanto para el paciente como para el profesional (12).

Los estudios antes mencionados hacen referencia a la importancia que tiene el uso de las técnicas de cementación en restauraciones indirectas a base de cerómeros, el propósito fue evaluar la resistencia a la tracción diametral de probetas in vitro de cementos dentales usados, BisCem, Duolink, Ketac Cem, Meron, Allcem, cuatro adhesivos convencionales y un autoadhesivo polimerizado químicamente como cementantes de puentes y coronas en prótesis fijas. 


\section{MATERIAL Y METODOS}

La investigación se realizó en el laboratorio del área de biomateriales de la facultad de Ing. Civil de la Universidad Andina del Cusco. Se midió la resistencia a la tracción diametral in vitro de 5 cementos: BisCem, Duolink, Ketac Cem, Meron y Allcem, sometidos a fotopolimerización y autopolimerizacion, con 20 especímenes para cada cemento.

Se tiene un diseño cuasi experimental, se confeccionaron 100 especímenes para los diferentes cementos de $8 \mathrm{~mm}$ de diámetro y $5 \mathrm{~mm}$ de alto, se utilizó un formador de probetas de polietileno de alta densidad estandarizado con las medidas antes mencionadas, el tipo de muestreo es no probabilístico. Para confeccionar cada cilindro, el formador fue aislado con vaselina siliconada y se agregó los diferentes cementos utilizados en un solo incremento, teniendo la precaución de que no queden poros o irregularidades en su superficie, se utilizó un porta-objeto haciendo presión sobre las caras libres del cilindro. Se polimerizó el material, utilizando una lámpara de fotocurado modelo XL3000, (3M U.S.A) por un lapso de 20 segundos en una superficie y 20 segundos más en la superficie opuesta, completando 40 segundos en total, la distancia de la lámpara para la polimerización se realizó aproximadamente a $2 \mathrm{~mm}$ de distancia entre el sitio de la cementación y la punta de guía de luz de la lámpara (12).

Los criterios de inclusión han sido: especímenes de prueba que cumplieron con las medidas establecidas; especímenes totalmente lisos y planos en su superficie sin defectos estructurales como líneas de fracturas y burbujas; especímenes previamente inspeccionados al detalle para evitar los defectos estructurales antes mencionados.

Las muestras identificadas fueron sometidas a una fuerza continua de $500 \mathrm{~kg}$, con una velocidad de desplazamiento fija de $1 \mathrm{~cm} / \mathrm{min}$. Dado que la magnitud de la carga soportada dependerá de la dimensión del cuerpo de prueba, para lograr valores equiparables a probetas de cualquier tamaño, se debe relacionar la carga ejercida con la dimensión del cuerpo de prueba, aplicando la siguiente formula:

$$
T s=\frac{2 \cdot P}{\pi \cdot D \cdot T}
$$

\section{Tensión a la tracción:}

Dónde:

$\mathrm{P}=$ Carga de fractura

$\mathrm{D}=$ Diámetro de la probeta

$\mathrm{T}=$ Espesor de la probeta

Reemplazando en la fórmula los valores de diámetro y espesor de las probetas obtenemos:

$$
\mathrm{T}=\frac{2 . P(k f g)}{\pi .8 \mathrm{~mm} \cdot 5 \mathrm{~mm}}
$$

Para transformar los valores a MPa reemplazamos en la fórmula los Kgf por Newton (N). Sabiendo que N $=9.8 \mathrm{Kgf}$ y $\mathrm{MPa}=\mathrm{N} / \mathrm{mm}^{2}$.

El pascal resulta ser una unidad pequeña para los estudios de resistencia a la tracción. Por ello se utiliza un múltiplo del mismo; el Mega pascal (MPa). Los resultados de la prueba mecánica fueron expresados y analizados por Análisis de Varianza ANOVA, comparando posteriormente los grupos entre sí. Las diferencias fueron consideradas significativas si el valor de probabilidad $(\mathrm{p})$ resulta inferior al $5 \%(\mathrm{p}<0,05)$ para establecer si se valida o rechaza la hipótesis planteada.

\section{RESULTADOS}

Se presenta la resistencia a la tracción diametral in vitro de cementos dentales de probetas en Megapascal (MPa), de 5 tipos de cemento dentales utilizados como cementantes de puentes y coronas en prótesis fijas con 20 ensayos en cada uno de un total de 100 especímenes (tabla 1), la resistencia promedio a la tracción diametral de probetas del cemento BisCem fue de 38,75 $\pm 11,56 \mathrm{MPa}$.

Al 95\% de confiabilidad, la resistencia a la tracción diametral in vitro del cemento dental BisCem que muestra un comportamiento normal de sus mediciones (Anderson - Darling: $\mathrm{p}=0,202>0,05$ ), está comprendido entre $33,344 \mathrm{MPa}$ hasta $44,165 \mathrm{MPa}$ (gráfico 1).

La resistencia promedio a la tracción diametral de probetas del cemento Duolink es de 64,30 MPa, con variación de $13.15 \mathrm{MPa}$, registrando un diámetro mínimo de 19,10 y máximo de 81,17 en este cemento dental (gráfico 2). 
Tabla 1. Resistencia a la tracción diametral in vitro de cementos dentales

\begin{tabular}{cccccc}
\hline Ensayo & $\begin{array}{c}\text { Bisem } \\
\left(\mathrm{N} / \mathrm{mm}^{2}\right)\end{array}$ & $\begin{array}{c}\text { Duolink } \\
\left(\mathrm{N} / \mathrm{mm}^{2}\right)\end{array}$ & $\begin{array}{c}\text { Ketac Cem } \\
\left(\mathrm{N} / \mathrm{mm}^{2}\right)\end{array}$ & $\begin{array}{c}\text { Meron } \\
\left(\mathrm{N} / \mathrm{mm}^{2}\right)\end{array}$ & $\begin{array}{c}\text { All Cem } \\
\left(\mathrm{N} / \mathrm{mm}^{2}\right)\end{array}$ \\
\hline 1 & 42,97 & 55,70 & 27,06 & 63,66 & 35,01 \\
2 & 28,65 & 69,10 & 39,79 & 55,70 & 55,70 \\
3 & 31,83 & 68,44 & 23,87 & 23,87 & 44,56 \\
4 & 14,32 & 66,85 & 55,70 & 111,41 & 46,15 \\
5 & 28,65 & 68,44 & 28,65 & 39,79 & 44,56 \\
6 & 35,01 & 76,39 & 27,06 & 68,44 & 55,70 \\
7 & 33,42 & 79,58 & 30,24 & 66,85 & 57,30 \\
8 & 41,38 & 68,44 & 33,42 & 55,70 & 62,07 \\
9 & 46,15 & 60,48 & 38,20 & 60,48 & 35,01 \\
10 & 55,70 & 58,89 & 39,79 & 50,93 & 44,56 \\
11 & 60,48 & 55,70 & 60,48 & 57,30 & 46,15 \\
12 & 28,65 & 68,44 & 58,89 & 68,44 & 55,70 \\
13 & 30,24 & 77,99 & 50,93 & 76,39 & 57,30 \\
14 & 35,01 & 81,17 & 38,20 & 73,21 & 54,11 \\
15 & 36,61 & 66,85 & 27,06 & 71,62 & 65,25 \\
16 & 44,56 & 68,44 & 28,65 & 60,48 & 57,30 \\
17 & 62,07 & 55,70 & 39,79 & 55,70 & 39,79 \\
18 & 42,97 & 60,48 & 50,93 & 23,87 & 44,56 \\
19 & 41,38 & 62,07 & 41,38 & 28,65 & 50,93 \\
20 & 35,01 & 66,85 & 44,56 & 30,24 & 54,11 \\
\hline
\end{tabular}

Al 95\% de confiabilidad, la resistencia a la tracción diametral in vitro del cemento dental Duolink que no muestra un comportamiento normal de sus mediciones (Anderson - Darling: $\mathrm{p}=0,005<0,05$ ), está comprendido entre 60,479 MPa hasta 68,437 Mpa.

La resistencia promedio a la tracción diametral de probetas del cemento Ketac Cem es de 32,23 $\mathrm{MPa}$, con variación de $11,33 \mathrm{MPa}$, registrando un diámetro mínimo de 23,87 y máximo de 60,48 en este cemento dental (gráfico 3).

Al 95\% de confiabilidad, la resistencia a la tracción diametral in vitro del cemento dental Ketac Cem que muestra un comportamiento normal de sus mediciones (Anderson - Darling: $p=0,139>0,05$ ), está comprendido entre 33,930 MPa hasta 45,533 $\mathrm{MPa}$.

La resistencia promedio a la tracción diametral de probetas del cemento Meron es de 57,14 MPa, con variación de $20,89 \mathrm{MPa}$, registrando un diámetro mínimo de 23,87 y máximo de 20,89 en este cemento dental (gráfico 4).

Al 95\% de confiabilidad, la resistencia a la tracción diametral in vitro del cemento dental Meron que muestra un comportamiento normal de sus mediciones (Anderson-Darling: $\mathrm{p}=0,107>0,05$ ), está comprendido entre 47,359 MPa hasta 66,914 Mpa.

La resistencia promedio a la tracción diametral de probetas del cemento Allcem es de 50,29 MPa, con variación de 8,49 MPa, registrando un diámetro mínimo de 35,01 y máximo de 62,25 en este cemento dental.

Al 95\% de confiabilidad, la resistencia a la tracción dia- 


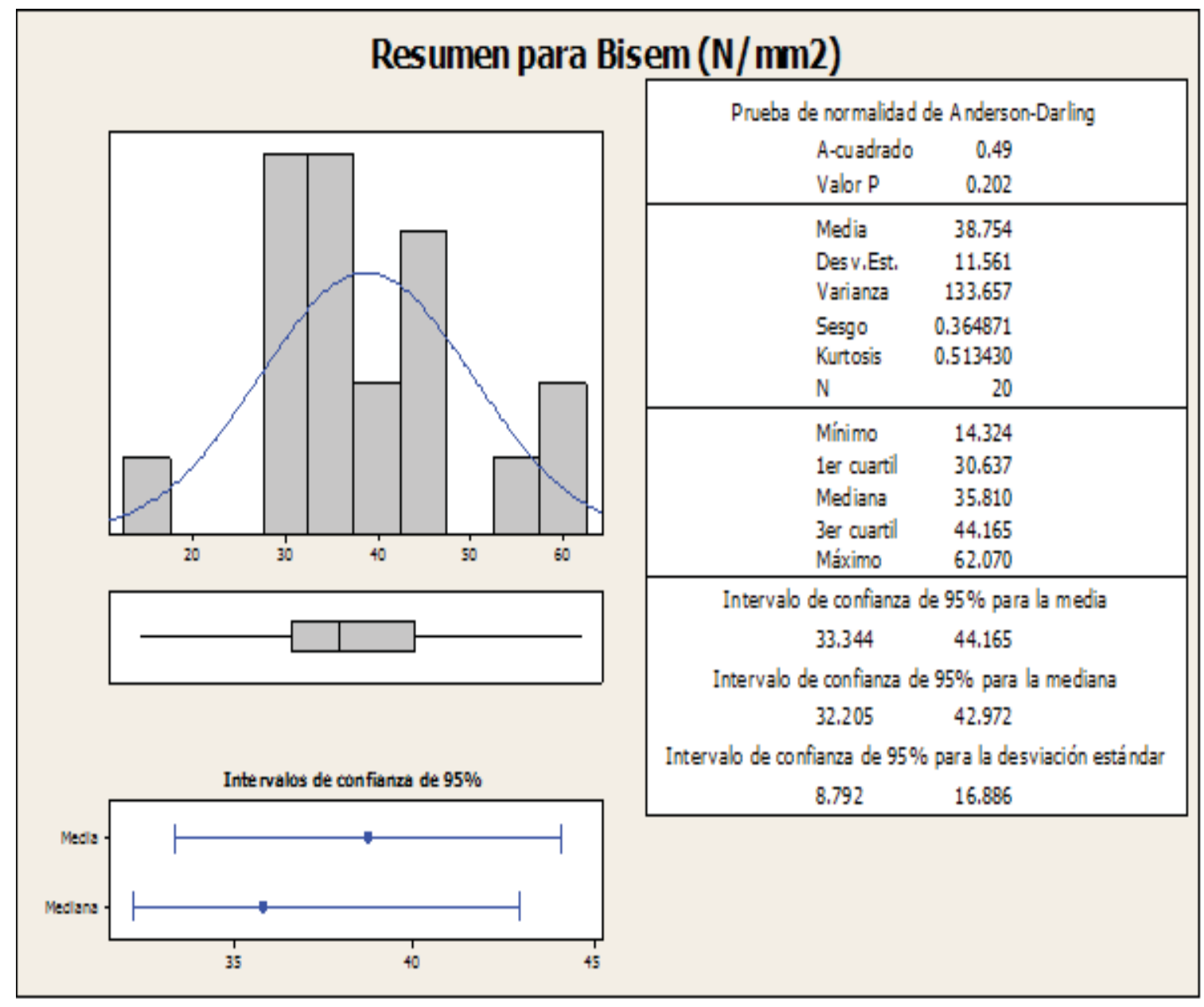

Gráfico 1 .Resistencia a la tracción diametral de probetas del cemento BisCem ${ }^{\circledR}$ 


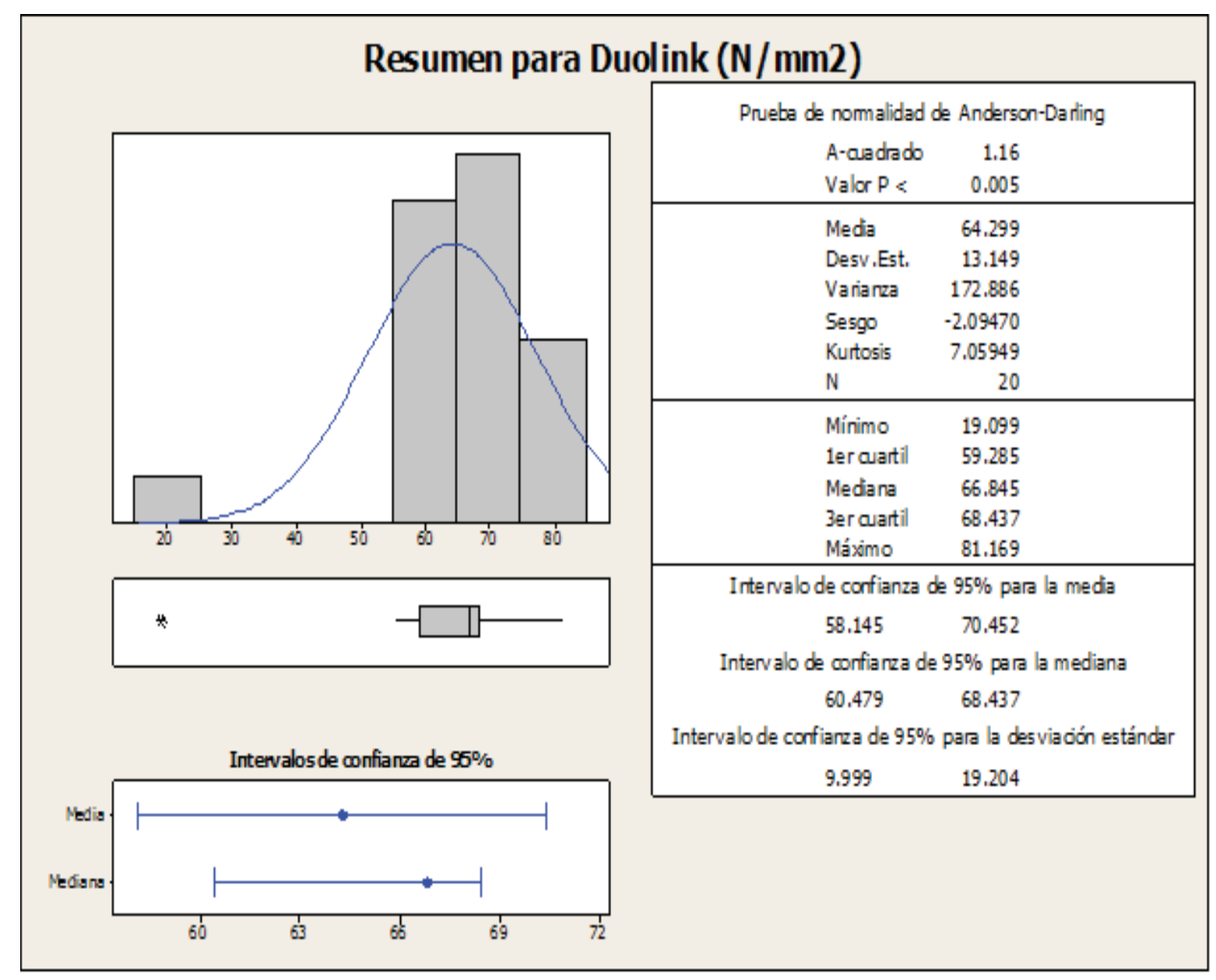

Gráfico 2.Resistencia a la tracción diametral de probetas del cemento Duolink ${ }^{\circledR}$ 


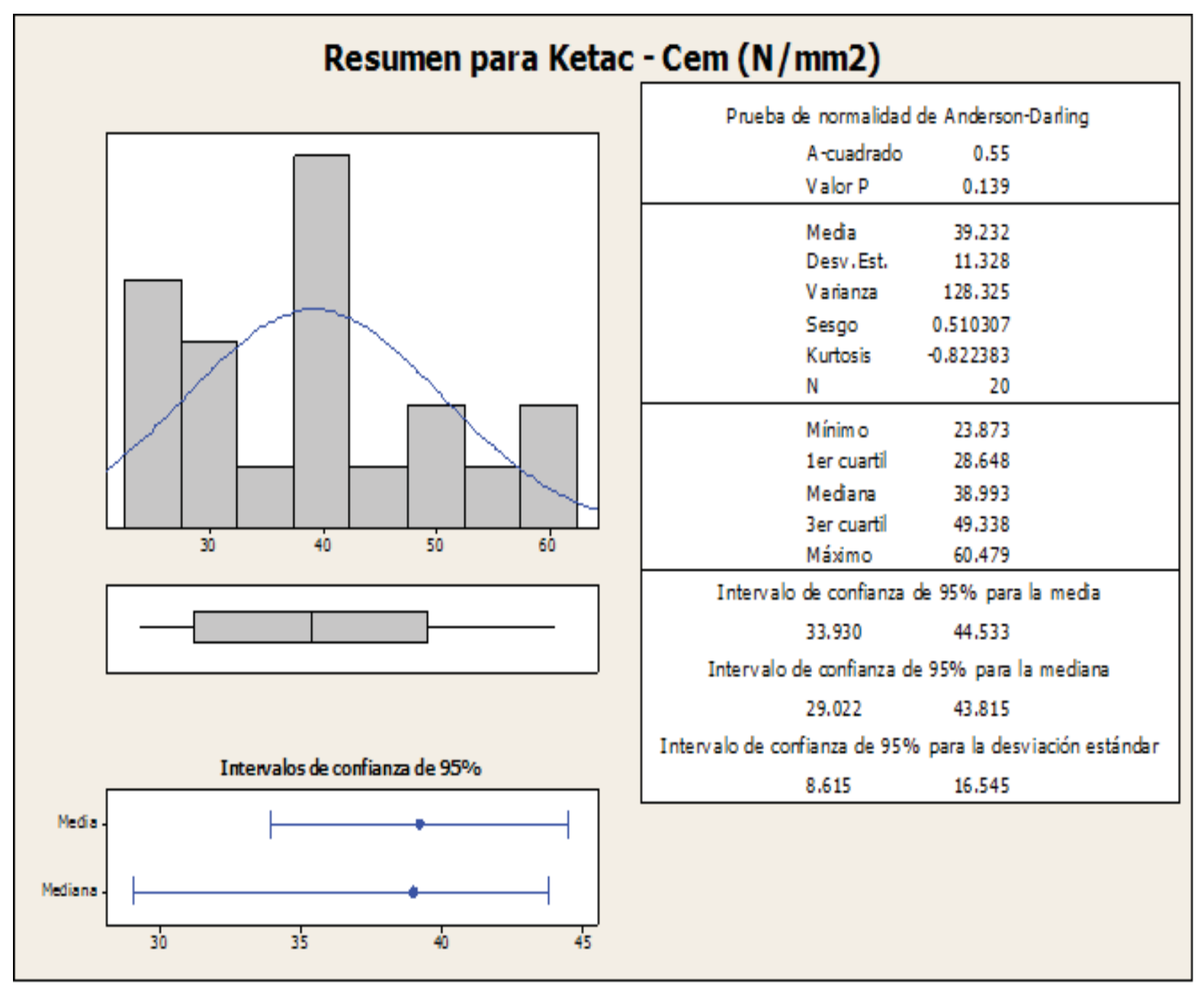

Gráfico 3.Resistencia a la tracción diametral de probetas del cemento Ketac Cem 


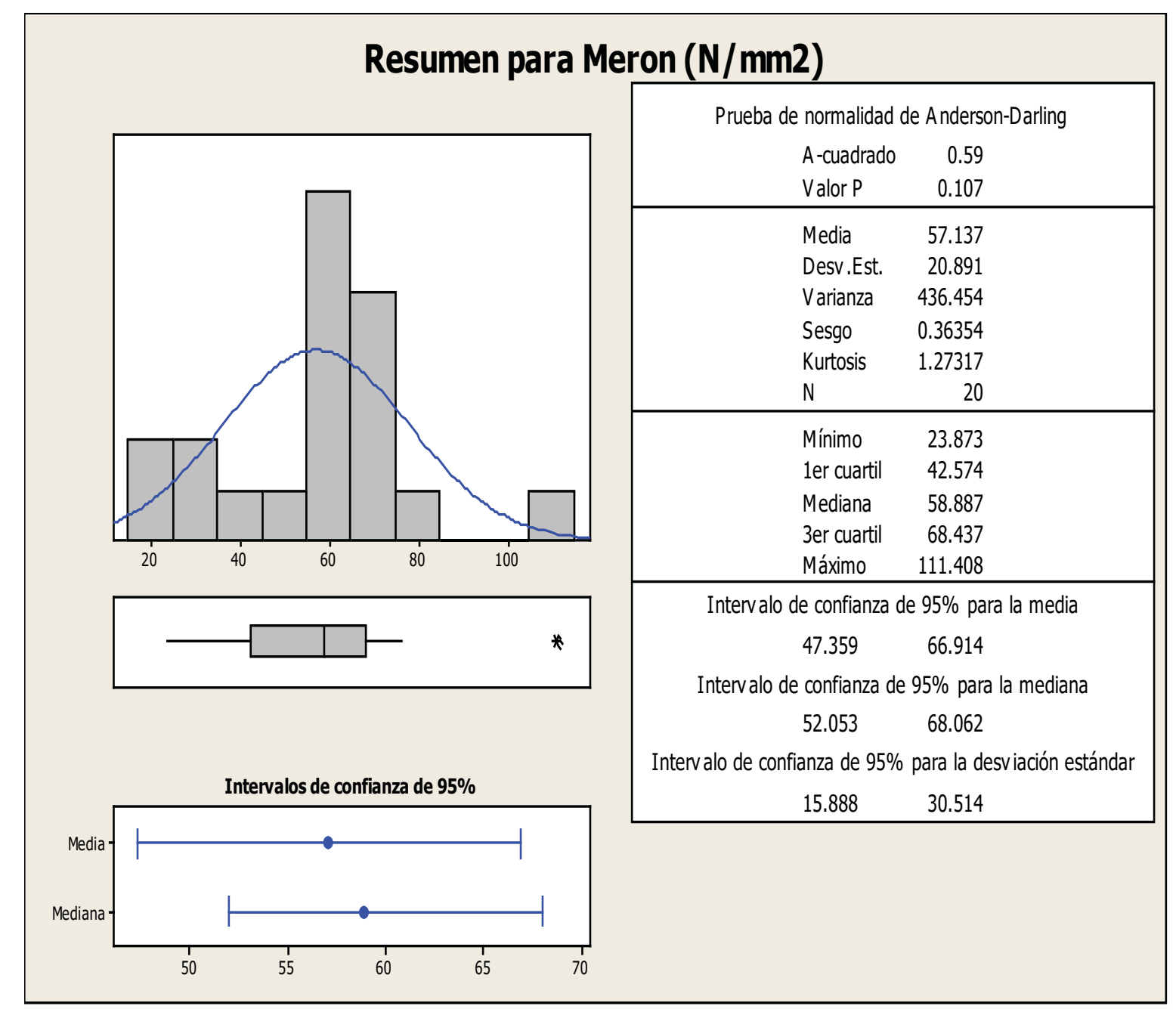

Gráfico 4. Resistencia a la tracción diametral de probetas del cemento Meron. 


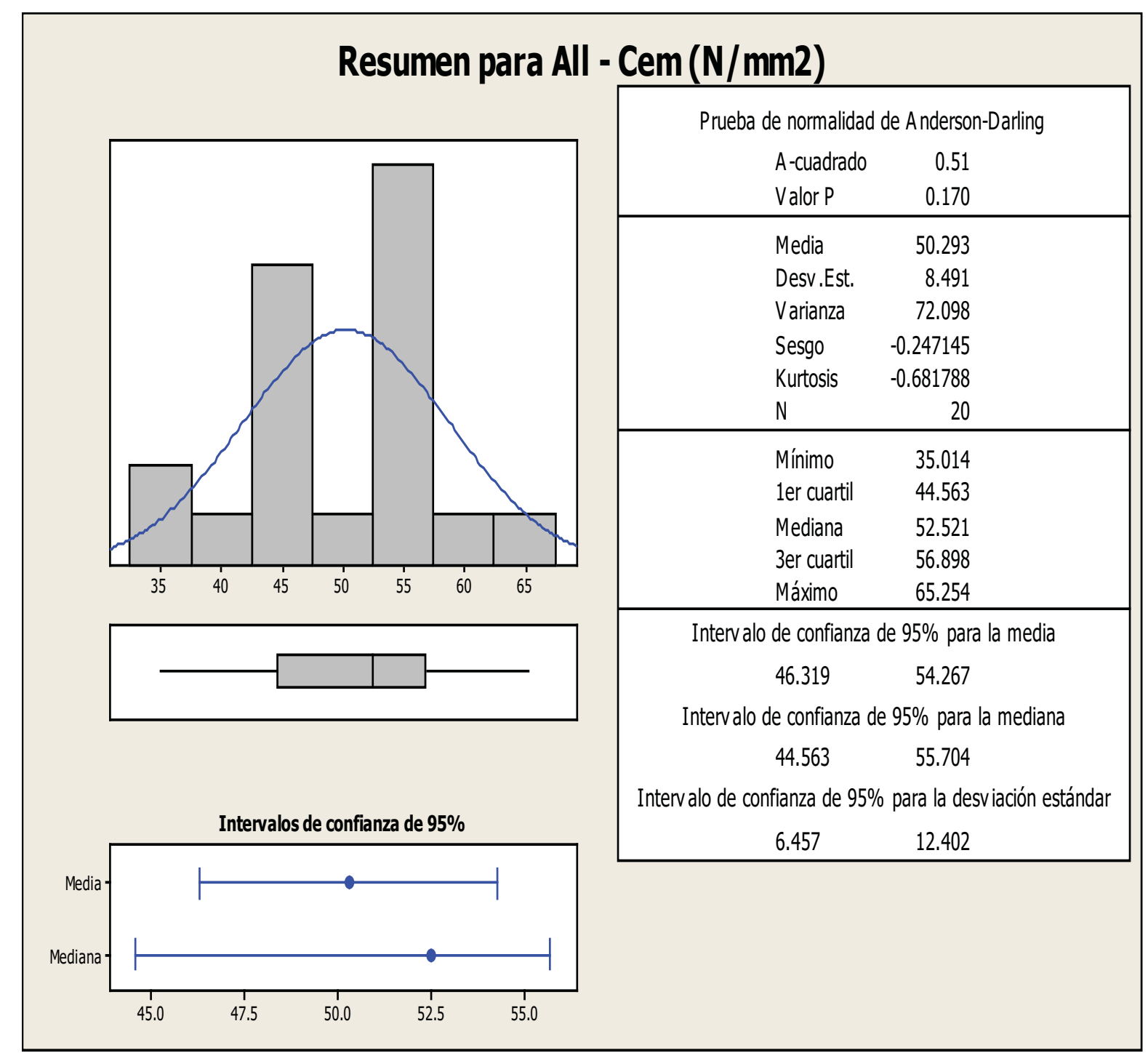

Gráfico 5. Resistencia a la tracción diametral de probetas del cemento All Cem. 
Tabla 2. Comparación de la resistencia a la tracción diametral in vitro de cementos dentales.

\begin{tabular}{|c|c|c|c|c|c|c|}
\hline \multirow[b]{2}{*}{ (I) Cementos } & \multirow[b]{2}{*}{ (J) Cementos } & \multirow{2}{*}{$\begin{array}{l}\text { Diferencia de } \\
\text { medias (I-J) }\end{array}$} & \multirow[b]{2}{*}{ Error típico } & \multirow[b]{2}{*}{ Sig. } & \multicolumn{2}{|c|}{ Intervalo de confianza al $95 \%$} \\
\hline & & & & & $\begin{array}{l}\text { Límite } \\
\text { inferior }\end{array}$ & $\begin{array}{l}\text { Límite } \\
\text { superior }\end{array}$ \\
\hline \multirow{4}{*}{ BisCem } & Duolink & $-25,54700^{*}$ & 434,388 & , 000 & $-376,267$ & $-134,673$ \\
\hline & Ketac Cem &,- 47950 & 434,388 & 1,000 & $-125,592$ & 116,002 \\
\hline & Meron & $-18,38350^{*}$ & 434,388 & , 001 & $-304,632$ & $-63,038$ \\
\hline & All Cem & $-1,153,800$ & 434,388 & 068 & $-236,177$ &, 5417 \\
\hline \multirow{4}{*}{ Duolink } & BisCem & $25,54700^{*}$ & 434,388 & , 000 & 134,673 & 376,267 \\
\hline & Ketac Cem & $25,06750^{*}$ & 434,388 &, 000 & 129,878 & 371,472 \\
\hline & Meron & 716,350 & 434,388 & , 470 & $-49,162$ & 192,432 \\
\hline & All Cem & $14,00900^{*}$ & 434,388 & ,015 & 19,293 & 260,887 \\
\hline \multirow{4}{*}{ Ketac - Cem } & BisCem & ,47950 & 434,388 & 1,000 & $-116,002$ & 125,592 \\
\hline & Duolink & $-25,06750^{*}$ & 434,388 &, 000 & $-371,472$ & $-129,878$ \\
\hline & Meron & $-17,90400^{*}$ & 434,388 & ,001 & $-299,837$ & $-58,243$ \\
\hline & All Cem & $-1,105,850$ & 434,388 & ,089 & $-231,382$ & 10,212 \\
\hline \multirow{4}{*}{ Meron } & BisCem & $18,38350^{*}$ & 434,388 & , 001 & 63,038 & 304,632 \\
\hline & Duolink & $-716,350$ & 434,388 & ,470 & $-192,432$ & 49,162 \\
\hline & Ketac Cem & $17,90400^{*}$ & 434,388 & ,001 & 58,243 & 299,837 \\
\hline & All Cem & 684,550 & 434,388 & ,516 & $-52,342$ & 189,252 \\
\hline \multirow{4}{*}{ All - Cem } & BisCem & $1,153,800$ & 434,388 & ,068 &,- 5417 & 236,177 \\
\hline & Duolink & $-14,00900^{*}$ & 434,388 & ,015 & $-260,887$ & $-19,293$ \\
\hline & Ketac Cem & $1,105,850$ & 434,388 & ,089 & $-10,212$ & 231,382 \\
\hline & Meron & $-684,550$ & 434,388 & ,516 & $-189,252$ & 52,342 \\
\hline
\end{tabular}

Comparaciones múltiples .Variable dependiente: resistencia.HSD de Tukey

* La diferencia de medias es significativa al nivel 0,05.

metral in vitro del cemento dental Allcem que muestra un comportamiento normal de sus mediciones (Anderson-Darling: $\mathrm{p}=0,170>0,05)$, está comprendido entre 46,319 MPa hasta 54,267 Mpa (gráfico 5).

Se observa que el cemento dental BisCem difiere significativamente respecto a la resistencia de tracción diametral (MPa) con el cemento dental Duolink y en cemento dental Meron. El cemento dental Duolink difiere significativamente con los cementos dentales BisCem y Ketac Cem. El cemento dental Ketac Cem difiere significativamente en cuanto a sus resistencias de los cementos dentales BisCem y Ketac Cem; y el cemento dental Allcem difiere significativamente del cemento dentales Duolink (tabla 2).

Se observa que el cemento BisCem $(38,753 \mathrm{MPa})$ y el cemento Ketac Cem (39.233 MPa) presentan menor resistencia a la tracción diametral de probetas; el cemento dental Duolink (64,3 MPa) es aquel que presenta mayor resistencia a la tracción diametral de probetas en comparación a Meron $(57,137 \mathrm{MPa})$ que es sus similares (gráfico 6). 


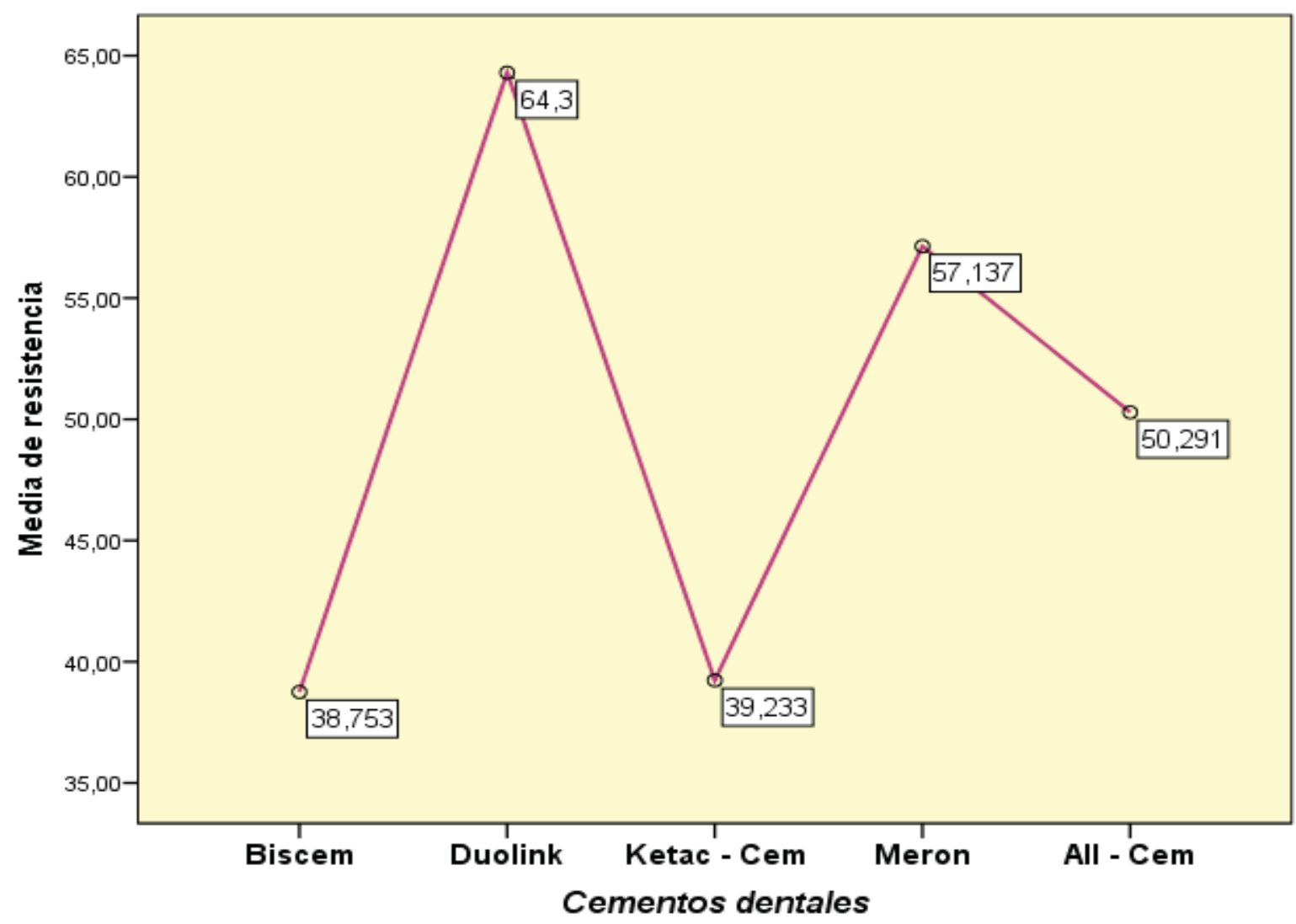

Gráfico 6.Comparación de la resistencia a la tracción diametral in vitro de cementos dentales

\section{DISCUSIÓN}

Actualmente, existen cementos resinosos autoadhesivos que, por su aplicación directa, sin necesidad de pre tratamiento del sustrato dental, constituyen una opción clínicamente atractiva, sin embargo, para su elección no debe dejar de considerarse sus propiedades físicas y químicas.

La adhesión se puede producir de diferentes formas tal como describen a continuación:

Dependientes de las superficies: Contacto íntimo, alta energía superficial, potencialmente receptivos a uniones químicas, superficie lisa vs rugosa.

Dependientes del biomaterial cementante o adhesivo: baja tensión superficial, alta capilaridad, multipotencialidad de enlace, alta estabilidad dimensional, alta resistencia mecánica, química adhesiva y cohesiva, fácil manipulación.
Es prudente limitar el tiempo de exposición de la resina al calor, por lo que debe ser precalentada solo la porción a ser utilizada en el momento de la cementación, lo cual debiese estar concertado y programado para dicha sesión (13).

La utilización de resina restaurativa como medio cementante, tiene como ventaja un margen de alto contenido de relleno, que no se desgasta, es fácil de retirar los excesos previo a su polimerización, capaz de rellenar cualquier pequeño defecto, de fácil de pulido y terminado. Pero con la desventaja de lograr obtener una capa de resina lo suficientemente fluida y delgada para no alterar su total asentamiento y así permitir su completa polimerización (9).

El ensayo de tracción de un material consiste en someter a una probeta normalizada aun esfuerzo axial de tracción creciente hasta que se produce la rotura de la probeta. Este ensayo mide la resistencia de un ma- 
terial a una fuerza estática o aplicada lentamente; las velocidades de deformación en un ensayo de tensión suelen ser muy pequeña.

Se ha evaluado la resistencia a la tracción hasta la rotura de la probeta en 5 cementos dentales: BisCem, Duolink, Ketac Cem, Meron y Allcem, sometidos a fotopolimerización y autopolimerizacion.

El proceso de fotopolimerización también puede causar una acumulación de calor significativa, in vitro e in vivo se reporta un aumento de temperatura intrapulpar de hasta $20^{\circ} \mathrm{C}$, dependiendo de la preparación del diente, el protocolo de fotoactivación y el material utilizado (14).

Barrancos en un estudio in vitro observó la resistencia a la fractura de coronas de cerámica de alúmina cementadas con tres tipos de cementos y diferentes técnicas de cementación, evaluandose la correlación existente entre los cementos y su resistencia a la fractura en las coronas libres de metal. En la actualidad las resinas compuestas constituyen uno de los materiales dentales de mayor uso y desarrollo en el ámbito odontológico a nivel mundial. Esto está directamente relacionado con sus cualidades estéticas y su facilidad de manipulación directa sobre las preparaciones cavitarias (15).

Bacchi refiere que las temperaturas que simula el proceso es de $5^{\circ} \mathrm{C}$ que representan los alimentos fríos que se consumen, $37^{\circ} \mathrm{C}$ que representa la temperatura habitual de la cavidad oral y $55^{\circ} \mathrm{C}$ que representa la de los alimentos calientes, los principales cambios observados son la hidrólisis del adhesivo, los cracks (fisuras) o fallos cohesivos y tags por percolación (16).

La distribución normal de los datos de los grupos en estudio es uno de los requisitos para poder utilizar el test inferencial paramétrico. Es por esto que el primer paso fue realizar una prueba de normalidad.

Debido a que el número de muestras es inferior a 50, el test utilizado es ShapiroWilk. En todos los grupos se obtuvo una distribución normal de los datos por lo que se prosiguió a realizar un análisis de varianza ANOVA, se acepta la hipótesis alternativa: existen diferencias significativas entre las propiedades físicas de los de cementos y entre las formas de activación.

La existencia de diferencias estadísticamente sig- nificativas entre los 5 cementos se debe a que estos cementos comparados BisCem-Duolink, son de tipo fotopolimerizables, pero el resultado que sorprende es la comparación de los cementos BisCem-Meron, debido a que el cemento Meron es autopolimerizable y aun así posee diferencias significativas debido quizá al uso del ácido policrilico y parabenos en su composición y a su alta biocompatibilidad con el tejido dentario. En cuanto a la no existencia de diferencias estadísticas con el cemento Ketac Cem debido a su auto polimerización y quizá por su fórmula granulada que hace que en el momento de mezclado no complete la homogenización, aún queda camino por recorrer para lograr un medio cementante ideal y foto polimerizar es una forma de mejorar, al menos levemente, las propiedades mecánicas de los cementos de resina.

Las características del material cerámico pueden determinar la cantidad de luz transmitida y el grado de polimerización de las resinas de cementación (17).

Una correcta adhesión proporciona alta retención, mejora la adaptación marginal, previene la microfiltración, y aumenta la resistencia a la fractura tanto del diente, como de la restauración (18).

Los cementos a base de resina dual, presentan tanto la polimerización inicial inducida por luz, como la auto polimerización. Cabe mencionar que, en este tipo de cementos, al igual que en las resinas, se observa el fenómeno de encogimiento por polimerización al realizar su endurecimiento $(19,20)$.

\section{CONCLUSIONES}

Existen diferencias estadísticamente significativas entre la resistencia a la tracción diametral de los 5 cementos dentales, siendo de mayor resistencia el Duolink seguido de Meron y Allcem que presentaron una resistencia a la tracción diametral promedio y por último se encontró a BisCem-Ketac Cem como los productos que obtuvieron menor resistencia a la tracción.

En las probetas del cemento de resina autoadhesiva Biscen se obtuvo una menor resistencia en cuanto a la tracción diametral. Las probetas de ionomeros de vidrio de cementación Ketac Cem, alcanzaron un mínimo de resistencia en relación a la tracción diametral. Las probetas del cemento de resina adhesiva Allcem presentaron una resistencia promedio en cuanto a la tracción diametral. 
Las probetas del cemento de resina adhesiva Duolink evidenciaron una mayor resistencia a la tracción diametral a diferencia de los demás. Las probetas de ionomero de vidrio Meron revelaron una resistencia promedio en cuanto a la tracción diametral.

\section{Correspondencia:}

Carolina Soto Carrión

Correo electrónico: caro7001@hotmail.com

\section{Declaración de financiamiento y de conflictos de interés:}

El estudio fue financiado por los autores.

Los autores declaran no tener conflictos de intereses.

\section{REFERENCIAS BIBLIOGRÁFICAS}

1. Polesel A. Restoration of the endodontically treated posterior tooth. G Ital Endod. 2014; 28:12-13.

2. Guimaraes IR, Gomez FM, De Goes MF. Effect of activation mode on 77 flexural strength and elasticity modulus of dual cure resin cements. Odovtos Int $\mathrm{J}$ Dent Sci. 2016;18(1):61-71.

3. Rivas V. Estudio comparativo in vitro de la tracción diametral y dureza superficial de resinas compuestas fluidas polimerizadas con lámpara halógena a través de bloques de Artglass (R). Tesis para título de Cirujano Dentista. Santiago de Chile: Universidad de Chile; 2012. 22-25 pp. Disponible en: http://www. repositorio.uchile.cl/handle/2250/117195 10/07/2016

4. White SN, Yu Z. Compressive and diametral tensile strengths of current adhesive luting agents. J Prosthet Dent. 1993; 69: 568-72. doi: 10.1016/00223913(93)90283-t

5. Ricaldi-Flores $\mathrm{C}$, Rengifo-Alarcon $\mathrm{C}$, Ricaldi-Flores J. Resistencia a la tracción de postes de fibra de vidrio cementados con resina autoadhesiva y resina de autocurado. Kiru. 2013; 10(1):26-31.

6. Furuichi T, Takamizawa T, Tsujimoto A, Miyazaki M, Barkmeier W, Latta M. Mechanical properties and sliding-impact wear resistance of self-adhesive resin cements. Oper Dent. 2016; 41(3):83-92. doi: 10.2341/15-033-L

7. Manso AP, Silnva N Bonfante E, Pegoraro T, Dias R, Carvalho R. Cements and adhesives for all-ceramic restorations. Dent Clin North Am. 2011; 55(2):31132. doi: 10.1016/j.cden.2011.01.011

8. Stamatacos C, Simon J. Cementation of indirect restorations: An overview of resin cements. AEGIS Communications. 2013; 34(1):42-46.

9. Van Dijken J, Pallesen U. Posterior bulk-filled resin composite restorations: A 5- year randomized controlled clinical study. J Dent. 2016; 51:29-35.
10. Palacio CF. Evaluación de la resistencia flexural de resinas compuestas precalentadas utilizadas como agente cementante en restauraciones indirectas. Tesis de especialista en Rehabilitación Oral. Quito, Ecuador: Universidad San Francisco de Quito; 2014.102-104 pp. Disponible en: http://repositorio.usfq.edu.ec/ handle/23000/3421 13/01/2016

11. Pozo A, García I. Estudio comparativo de tres sistemas de cementación para cerómeros. Tesis de Bachiller. Quito: Universidad Central del Ecuador; 2015. Disponible en: http://www.dspace.uce.edu.ec/ handle/25000/3766 13/01/2016

12. Bucheli M. Efecto en la integridad dental y en la de materiales restauradores producido por la acción de diferentes cepillos dentales en combinación con dentífricos de distinta abrasividad Tesis de Grado para Especialista en Rehabilitacion Oral. Quito, Ecuador: Universidad San Francisco de Quito; 2017. 118-119 pp.

13. Luna T. Estudio Comparativo in vitro: Resistencia a la tracción del Cemento resinoso dual y la resina compuesta precalentada como agente cementante en restauraciones indirectas a base de Cerómeros. Tesis de Maestria. Quito: Universidad Central del Ecuador; 2017.46-48 pp.

14. Wagner W, Aksu M, Neme A, Linger J, Rosa F, Walker S. Efecto del precalentamiento compuesto de resina de microfiltración restauración. Oper Dent. 2008; 33: 72-78.

15. Barrancos J. Operatoria Dental. Buenos Aires. Editorial Medica Panamericana; 2006.p. 55-60.

16. Bacchi A, Abuna G, Consani RL, Sinhoreti B, Sauro $\mathrm{S}$, Feitosa V. Effects of simulated pulpal pressure, mechanical and thermo cycling challenge on the microtensile bond strength of resinluting cements. Inter J Adhesion Adhesives. 2015; 60:69-74.

17. Rasetto FH, Driscoll CF, von Fraunhofer JA. Effect of light source and time on the polymerization of resin cement through ceramic veneers. J Prosthodont. 2001; 10(3):133-9. doi: 10.1111/j.1532-849x.2001.00133.x

18. Nuray TM. Mechanical and phisical properties of contemporary dental luting agents. J Prosther Dent. 2003; 89(2):127-34.

19. Hulsmann M, Heckendorff M, Lennon A. Chelating agents in root canal treatment mode of action and indications for their use. Int Endod J. 2003;36(12):81030. doi: 10.1111/j.1365-2591.2003.00754.x

20. Simancas Y, Rosales J, Vallejo E. Efecto del termociclado y el acondicionamiento en el sellado de una resina microhíbrida. AOV. 2012; 50(2):12-16.

Recibido: 20-12-19

Aceptado: 15-04-20 